\title{
Avaliação ultra-sonográfica do sistema urinário, fígado e útero do macaco-prego, Cebus apella ${ }^{1}$
}

\author{
Flávio R. Alves ${ }^{2 *}$, Francisco B. Costa ${ }^{3}$, Mirela M.S. Arouche ${ }^{4}$, Ana C.E. Barros ${ }^{3}$, Maria \\ A. Miglino ${ }^{5}$, Luiz C. Vulcano ${ }^{6}$ e Porfírio C. Guerra ${ }^{7}$ \\ ABSTRACT.- Alves F.R., Costa F.B., Arouche M.M.S., Barros A.C.E., Miglino M.A., Vulcano L.C. \& \\ Guerra P.C. 2007. [Ultrasonographic evaluation of the urinary system, liver and uterus of \\ Cebus apella monkey.] Avaliação ultra-sonográfica do sistema urinário, fígado e útero do \\ macaco-prego, Cebus apella. Pesquisa Veterinária Brasileira 27(9):377-382. Departamento de \\ Cirurgia, Faculdade de Medicina Veterinária e Zootecnia, Universidade de São Paulo, Av. Prof. \\ Dr. Orlando Marques de Paiva 87, São Paulo, SP 05508-270, Brazil. E-mail: flaviovet@usp.br \\ The Brown Capuchin, Cebus apella, has a wide distribution in the northern and southern \\ Brazilian Amazon region and in the Cerrado (savanna). These monkeys are usually submitted \\ to predatory chase, increasing the need for preservation of this wild animal species. An \\ ultrasonographic examination of 10 Brown Capuchins was made in order to describe the normal \\ ultrasonographic anatomy of their abdominal cavity. The urinary bladder revealed its wall \\ thickness with an average of $0.2 \mathrm{~cm}$, the topographic situation of which allowed close relation \\ with the wall of uterus and descendent colon. Using caudal abdominal scan, images of aorta, \\ caudal vena cava and right iliac vein were obtained. Liver was accessible for examination by \\ sagittal and cross-section ultrasound, allowing visualization of gallbladder and hepatic vessels. \\ Renal scan allowed accuracy to evidence the echogenicity differences between pelvis, renal \\ sinus, as well as the cortical-medullary relationship. The mean length of the kidneys was \\ $6.24 \pm 0.31 \mathrm{~cm}$, and no significant differences were observed between left and right kidney \\ length (Student's $t$-test and ANOVA). The renal volume obtained was $2.37 \pm 0.18 \mathrm{~cm}^{3}$. Correlation \\ Coefficients of Pearson between right and left renal length and between right and left renal \\ volume were $r=0.74$ and 0.51 . Mean thickness for cortical and medullar regions was $0.75 \pm 0.11$ \\ and $0.39 \pm 0.06 \mathrm{~cm}$, respectively. Correlation Coefficient of corticomedullar relation between \\ right and left renal was $r=0.19$. Examination by ultrasound was considered an efficient, non- \\ invasive, fast and repeatable technique which provides useful data for clinicians and surgeons \\ engaged in wild animal medicine.
}

INDEX TERMS: Cebus apella, Brown Capuchin, ultrasound, wild animals, Amazon.

\footnotetext{
${ }^{1}$ Recebido em 6 de junho de 2007.

Aceito para publicação em 14 de setembro de 2007.

2 Pós-Graduando em Anatomia dos Animais Domésticos e Silvestres, Departamento de Cirurgia, Faculdade de Medicina Veterinária e Zootecnia (FMVZ), Universidade de São Paulo (USP), Av. Prof. Dr. Orlando Marques de Paiva 87, Cidade Universitária, São Paulo, SP 05508-270, Brasil. *Autor para correspondência: flaviovet@usp.br

${ }^{3}$ Pós-Graduando em Ciências Veterinárias do Curso de Medicina Veterinária (CMV), Universidade Estadual do Maranhão (UEMA), Cidade Universitária Paulo VI, Tirirical, Cx. Postal 9, São Luís, MA 65055-970, Brasil.

${ }^{4}$ Médica Veterinária autônoma, Rua Edmundo Calheiros 795, São Luís, MA 65075-390, Brasil.

${ }^{5}$ Departamento de Cirurgia, FMVZ, USP, São Paulo, SP, Brasil.

${ }^{6}$ Departamento de Radiologia e Reprodução Animal, Universidade Estadual Paulista (Unesp), Rua Tiradentes 67, Botucatu, SP 18611-840, Brasil.

${ }^{7}$ Departamento de Clínicas, CMV, UEMA, São Luís, MA, Brasil.
}

RESUMO.- O macaco-prego, Cebus apella, é muito difundido no norte e sul da Amazônia Legal Brasileira e no Cerrado. Estes animais encontram-se rotineiramente submetidos à caça predatória, aumentando assim a necessidade de preservação desta espécie silvestre. Realizou-se um estudo ultra-sonográfico de 10 macacos-prego como forma de descrever a anatomia ultra-sonográfica normal de sua cavidade abdominal. A vesícula urinária apresentou parede com espessura média $0,2 \mathrm{~cm}$ e em posição anatômica cuja topografia permitiu contato com as paredes do corpo do útero e cólon descendente. À varredura abdominal caudal foi visualizada a aorta, veia cava caudal e veia ilíaca direita. O fígado foi visto em varredura sagital e transversal, possibilitando a observação da vesícula biliar e vasos hepáticos. A varredura renal demonstrou com 
precisão a pelve, seio renal e relação cortico-medular. 0 comprimento médio de ambos os rins foi de $6,24 \pm 0,31 \mathrm{~cm}$, não existindo diferença estatística entre o rim direito e esquerdo (Teste $t$ de Student e ANOVA). O volume renal foi $2,37 \pm 0,18 \mathrm{~cm}^{3}$. Os coeficientes de Correlação de Pearson entre os comprimentos renais direito e esquerdo e entre volumes renais direito e esquerdo foram dispostos como $r=0,74 \mathrm{e}$ 0,51 . As espessuras médias para a região cortical e medular foram $0,75 \pm 0,11 \mathrm{~cm}$ e $0,39 \pm 0,06 \mathrm{~cm}$, respectivamente. 0 coeficiente de correlação para a relação cortico-medular entre os rins direito e esquerdo foi de $r=0,19$. O exame ultrasonográfico mostrou-se como uma técnica eficiente, nãoinvasiva, rápida e reprodutível, que provê dados importantes aos profissionais da área de clínica e cirurgia de animais silvestres.

TERMOS DE INDEXAÇÃO: Cebus apella, macaco-prego, ultra-som, animais silvestres, Amazônia.

\section{INTRODUÇÃO}

O macaco-prego (Cebus apella) é difundido tanto ao norte quanto ao sul da Amazônia Legal Brasileira e no Cerrado, locais que sofrem rotineiramente intensa intervenção humana. A destruição progressiva de áreas verdes, habitat natural desses animais, os tem levado a migrarem para outras regiões, tornando-os, assim, mais susceptíveis à caça predatória.

A necessidade de preservação das espécies silvestres traz consigo a exigência de um maior conhecimento anatômico e clínico para estes animais. A criação em cativeiro já se constitui uma realidade, contudo, pouco foi discutido sobre a utilização de técnicas minimamente invasivas de investigação para avaliação de órgãos e estruturas abdominais nestes animais.

Em primatas não-humanos, dados referentes a esse estudo encontram-se restritos às avaliações feitas por James et al. (1976) e trabalhos que avaliaram o trato reprodutivo. Nenhuma referência foi encontrada para o macaco-prego, valendo a pena ressaltar que a anatomia de Cebus apella não é igualmente conhecida como em outros macacos, talvez pela sua restrição ao continente Sul-Americano.

Nesse contexto, a avaliação ultra-sonográfica da cavidade abdominal do macaco-prego conduz à possibilidade da caracterização da aparência sonográfica de órgãos e estruturas abdominais para esta espécie, gerando ferramentas necessárias para o tratamento clínico e cirúrgico, contribuindo para sua conservação, enquanto espécie silvestre.

\section{MATERIAL E MÉTODOS}

\section{Animais}

Foram utilizados 10 macacos-prego (Cebus apella), oriundos do Centro de Triagem de Animais Silvestres (CETAS) do Município de São Luís, Maranhão. Os animais foram agrupados de forma aleatória e à disponibilidade de chegada ao CETAS, sendo assim distribuídos em dois grupos, compostos por 6 machos e 4 fêmeas.

\section{Procedimento anestésico}

Os animais foram submetidos à contenção química utilizando acepromazina a $1 \%$, um fenotiazínico de ação central, na dosagem de $0,1 \mathrm{mg} / \mathrm{kg}$, por via intramuscular, aguardando-se 20 minutos até 0 início da manipulação dos animais. Após esta fase, foi realizada tricotomia ampla da região abdominal, tomando-se como limite cranial o último par de costelas e cartilagem xifóide; os processos transversos das vértebras lombares como limite lateral e; caudalmente a região inguinal em toda sua extensão. Seguiu-se o protocolo anestésico utilizando-se cloridrato de quetamina a $5 \%$ na dosagem de $11 \mathrm{mg} / \mathrm{kg}$ associado ao diazepan, na dosagem de $0,25 \mathrm{mg} /$ $\mathrm{kg}$, por via intramuscular, iniciando-se o exame ultra-sonográfico após 15 minutos. A associação utilizada neste protocolo promoveu um tempo hábil anestésico médio de 45 minutos em todas as avaliações, não havendo, portanto, necessidade de reaplicações sucessivas de drogas durante os exames.

\section{Exame ultra-sonográfico}

Procedeu-se o estudo ultra-sonográfico através de varredura abdominal para visualização e caracterização de suas estruturas. Para isso, utilizou-se um aparelho de ultra-som portátil PIE MEDICAL Falco Vet. e um transdutor convexo multifreqüencial, cuja frequiência variava entre 5 e 7,5 MHz. Como meio de contato para transmissão de ultra-sons foi utilizado o Carbogel ${ }^{\circledR}$.

Os animais foram posicionados na mesa de exames em decúbito dorsal com os membros torácicos e pélvicos levemente afastados, sem a necessidade de qualquer outro tipo de contenção, além da contenção química previamente realizada.

O exame foi iniciado pela região inguinal, utilizando-se a vesícula urinária como janela acústica. $\mathrm{O}$ seu estudo foi realizado com o animal disposto em decúbito dorsal, sendo estudada através de cortes sagitais e transversais. Para as fêmeas, procedeu-se a varredura da região abdominal caudal e cavidade pélvica, buscando avaliar a topografia e sintopia do corpo do útero. O exame prosseguiu deslocando-se o transdutor cranialmente, através de um acesso lateral direito para a visualização da aorta abdominal, na origem da bifurção das artérias ilíaca direita e esquerda, a partir das quais se prosseguiu a varredura em curso cranial para caracterização da artéria renal direita e esquerda para o exame de cada rim.

O fígado foi visualizado através de varredura sagital e transversal da linha mediana e seguindo todo o curso do último par de costelas. O parênquima renal foi acessado seguindo o curso da aorta até a identificação da artéria renal e seu hilo, onde foram realizados cortes sagitais e transversais e tomadas medidas de comprimento, largura e espessura da cortical e medular, bem como o volume.

As imagens foram documentadas e processadas através do sistema de captura de imagens do aparelho de ultra-som e os achados submetidos à análise estatística através do programa BioEstat 3.0.

\section{RESULTADOS}

Em corte sagital, a vesícula urinária foi visualizada como uma estrutura globosa localizada na transição pélvico-abdominal, ventralmente ao cólon descendente e em íntima relação com o corpo do útero, nas fêmeas (Fig.1a).

A vesícula urinária mostrou-se repleta, o que facilitou o seu fácil acesso. Em todos os exames, apresentou conteúdo anecóico e paredes hiperecóicas, com espessura média de $0,2 \mathrm{~mm}$. Em corte transversal, as fêmeas apresentaram o corpo do útero como uma estrutura circular, densa e de característica hipoecóica, comprimindo a vesícula urinária dorsoventralmente, medindo em média $1,71 \mathrm{~cm}$ de diâmetro. O cólon descendente, repleto de gás e de aparência hiperecóica, mostrou-se localizado dorsolateralmente à es- 

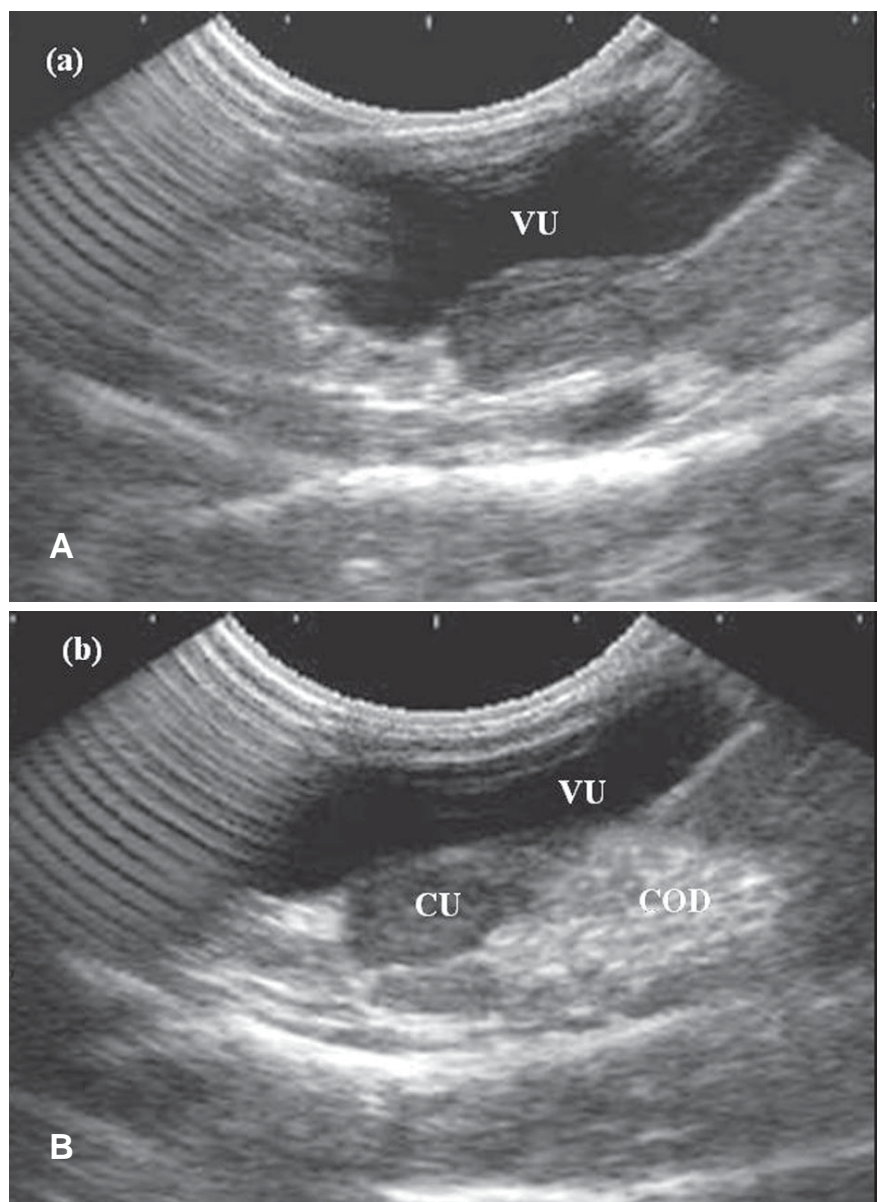

Fig.1. Imagem ultra-sonográfica de varredura transversal da vesícula urinária. Em (a) a relação anatômica entre o corpo do útero (CU) e a vesícula urinária (VU). Em (b) a relação de sintopia entre a vesícula urinária, corpo do útero e cólon descendente (COD).

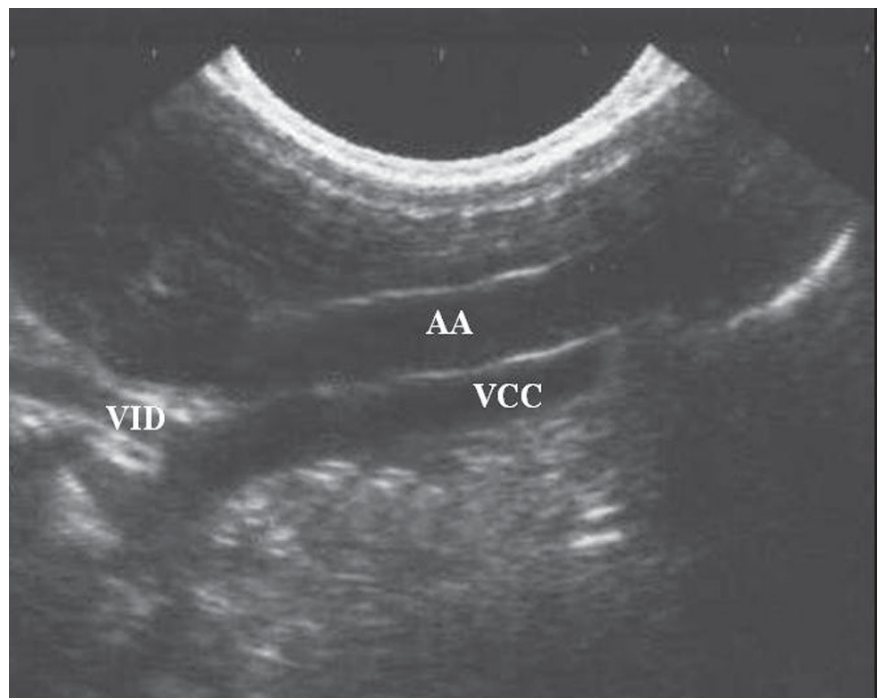

Fig.2. Imagem ultra-sonográfica de varredura sagital abdominal caudal, demonstrando a aparência ultra-sonográfica da aorta (AA) e veia cava caudal (VCC). Observa-se nesse ponto a veia cava caudal recebendo a veia ilíaca direita (VID).
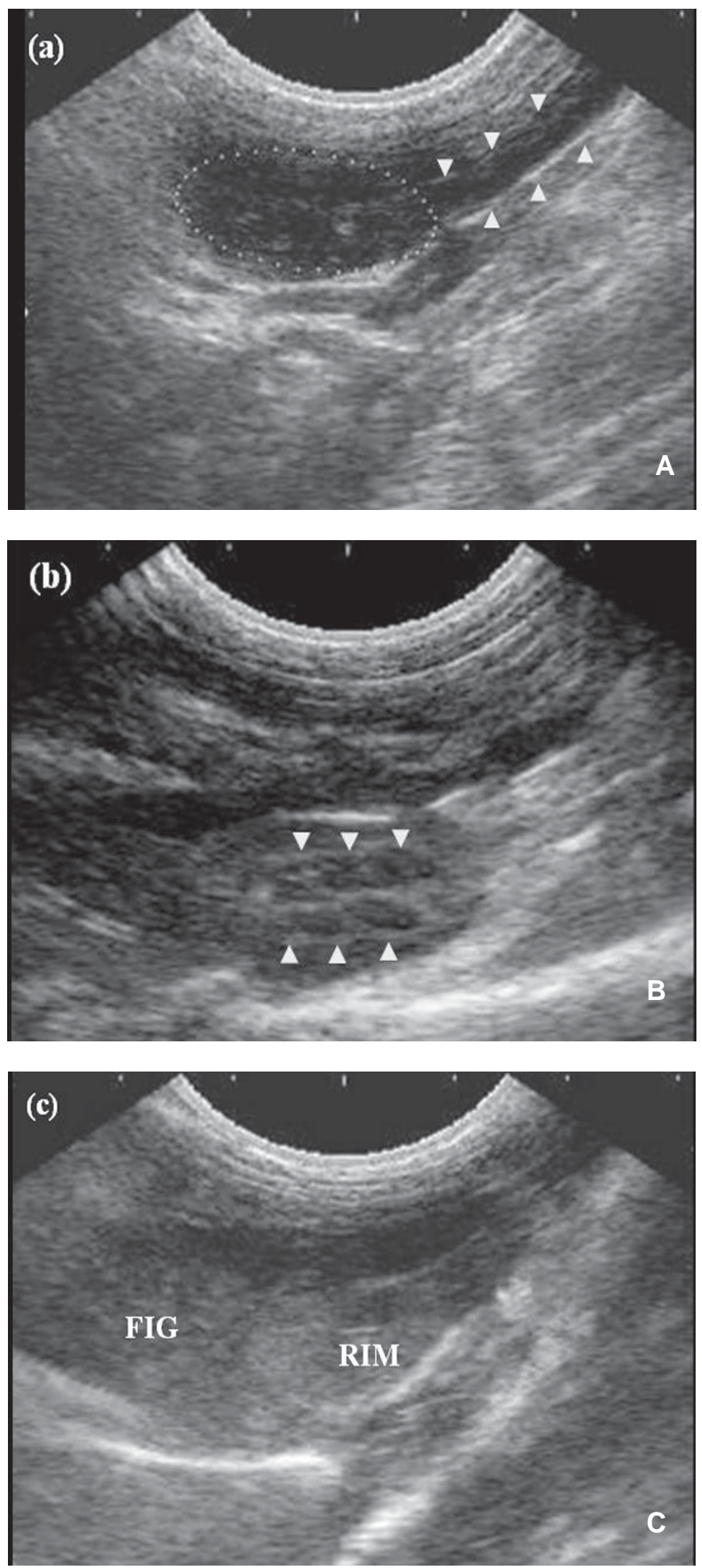

Fig.3. Imagem ultra-sonográfica de varredura sagital abdominal cranial, demonstrando a aparência ultra-sonográfica do fígado. (a) Nota-se sua arquitetura parenquimal grosseira e ecogeneicidade média e a vesícula biliar repleta. (b) Observam-se as veias porta (setas) de menor calibre e paredes hiperecogênicas, quando comparadas as veias hepáticas, vistas tanto em corte transversal quanto sagital. (c) Ponto onde a veia cava caudal recebe a veia porta, próximo ao diafragma. 


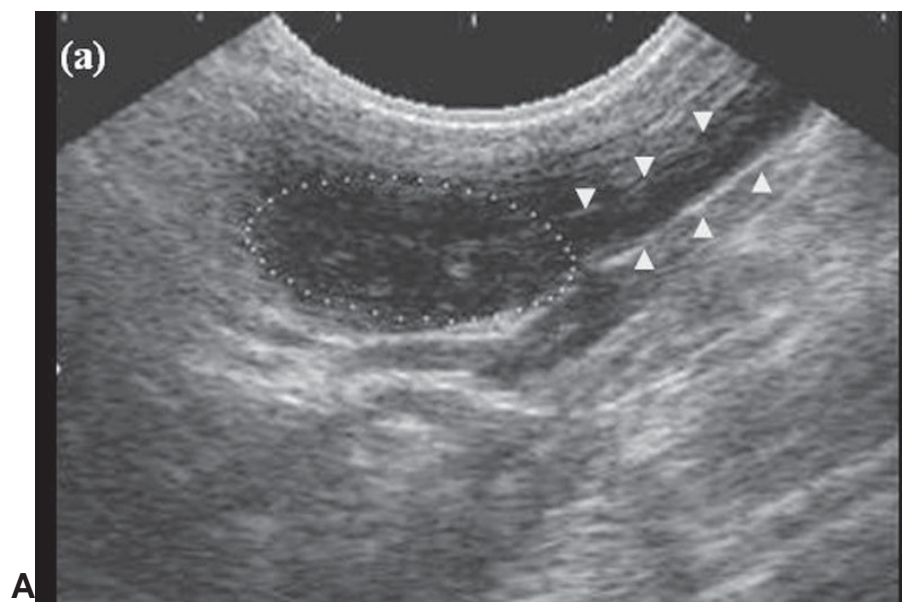

Fig.4. Imagem ultra-sonográfica de varredura sagital do rim. A artéria renal (setas) é vista caudalmente ao pólo renal orientando-se em direção ao seu hilo. Em (b) Observa-se uma região central hiperecóica caracterizada pela pelve e seio renal. A região medular hipoecóica é vista ao redor do seio renal, contrastando com a região cortical, mas hiperecóica. É notório o sinal de margem formado na transição córtico-medular, formado pela deposição gordura regional. Em (c) a comparação entre cortical medular e parênquima hepático, revelando ecogenicidade renal levemente maior.

querda, em relação ao corpo do útero e à vesícula urinária (Fig.1b).

A aorta foi visualizada com diâmetro de $1,17 \mathrm{~cm}$. Acompanhando seu curso observou-se a veia cava caudal recebendo a veia ilíaca direita, orientada caudolateralmente. A aorta pode ser diferenciada da veia cava caudal por apresentar parede mais espessa e hiperecogênica (Fig.2).

O figado foi observado ocupando toda a extensão do abdome cranial, em íntimo contato com a cúpula diafragmática. Ao exame ultra-sonográfico caracterizou-se como um órgão de arquitetura parenquimal grosseira, de ecogenicidade média (Fig.3a). Evidenciou-se a vesícula biliar repleta por conteúdo anecóico.

As veias porta puderam ser bem visualizadas, com paredes apresentando-se hiperecogênicas, diferenciando-as das veias hepáticas, presentes no mesmo plano (Fig.3b). A veia cava caudal pode ser vista próximo ao diafragma para receber as veias hepáticas (Fig.3c).

A avaliação renal foi realizada seguindo o curso da aorta até a identificação da artéria renal e seu hilo (Fig.4a). Anatomicamente, os rins apresentaram formato elíptico, com o rim direito posicionado mais cranialmente, quando comparado ao esquerdo. Ao corte longitudinal, a cápsula renal aparece bem distinta, levemente hiperecóica, quando o transdutor é posicionado perpendicularmente. A região cortical mostrouse mais ecogênica, quando comparada à região medular, onde estão presentes túbulos coletores, promovendo maior acúmulo de fluidos, tornando-a mais hipoecogênica. A região central, formada pelo seio e pelve renal, foi observada mais hiperecogênica, principalmente devido a maior quantidade
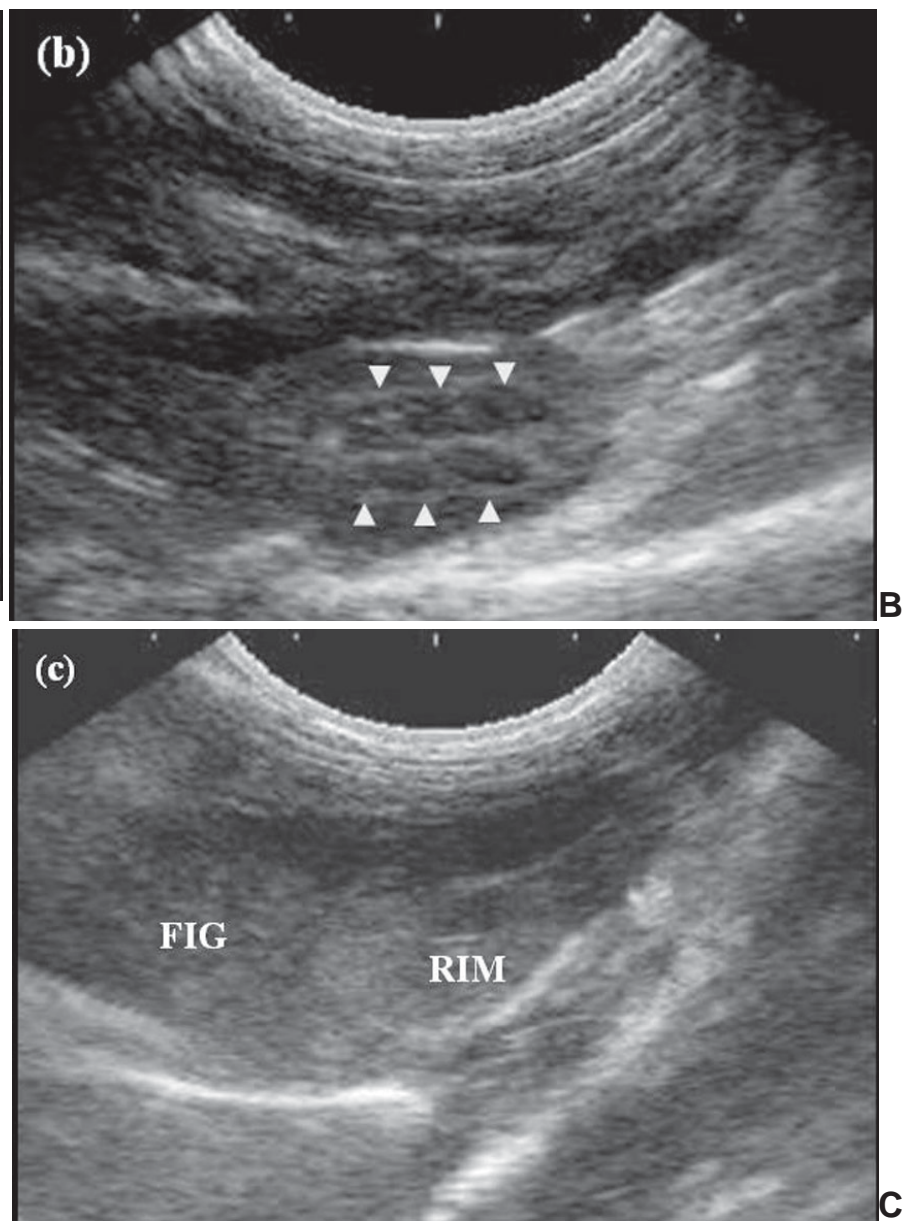

de gordura pélvica e peripélvica (Fig.4b). A relação de ecogenicidade, quando se comparou o parênquima renal e hepático, revelou ecogenicidade levemente maior para a cortical renal e em alguns momentos não houve diferença perceptível. É notório o sinal de margem na transição corticomedular formado pela deposição de gordura regional (Fig.4c).

A média de comprimento para ambos os rins foi de $6,24 \pm 0,31 \mathrm{~cm}$, não se identificando diferença estatística significante entre os comprimentos dos rins direito e esquerdo, conforme observado através do Teste $t$ de student ( $t$ crítico $=2.26 ;$ t calculado $=1,28)$ e ANOVA $(F=0,47 ; p=0,51)$. Contudo, observou-se alta correlação entre os comprimentos renais direito e esquerdo, evidenciado através da Correlação de Pearson $(r=0,74)$ e uma correlação média para os volumes $(r=0,51)$. Não foi observada correlação entre as regiões cortical e medular $(r=0,19)$. A relação cortico-medular foi de aproximadamente 2:1, mantendo-se constante durante todas as avaliações. As espessuras médias para a região cortical e medular foram respectivamente, $0,75 \pm 0,11$ e $0,39 \pm 0,06 \mathrm{~cm}$. $\mathrm{O}$ volume renal médio observado foi de $2,37 \pm 0,18 \mathrm{~cm}^{3}$.

\section{DISCUSSÃO}

Utilizando-se um transdutor 7,5 MHz é possível obter uma adequada resolução para o estudo da cavidade abdominal de Cebus apella. Um protocolo anestésico é necessário para 
minimizar o estresse, bem como evitar riscos de injúrias, auxiliando assim a otimização do exame.

Para este estudo, o exame ultra-sonográfico promoveu boa resolução de imagem para a vesícula urinária, corpo do útero, fígado e os rins. Também foi possível o estudo topográfico de grandes vasos como aorta, veia cava caudal e veia ilíaca direita. A topografia e tamanho esplênico não permitiram visualização detalhada do baço na maioria dos exames, não sendo tecidas observações sobre este órgão neste trabalho.

A identificação da vesícula urinária provavelmente é dependente de sua característica de preenchimento. Em nossas avaliações, esta se mostrou repleta em todos os exames realizados, contrapondo-se as informações encontradas em estudos realizados com Marmoset (Callithrix jacchus), onde a vesícula urinária foi mais comumente observada em fêmeas (Wagner et al. 2005).

Nas espécies domésticas como o cão e o gato, a avaliação uterina pode ser melhor realizada quando em gestação ou em estro (Matton \& Nyland 2002). Contudo, todas as fêmeas observadas apresentaram boa visualização do corpo do útero, no entanto, sem demonstrar características de receptividade sexual em relação ao macho, constituindo-se esse achado provavelmente uma característica inerente à espécie, como também é observado na espécie humana (Bates 2004).

Tal qual as observações realizadas em estudos humanos (Bluth 1984, Cerri \& Rocha 1993, Coelho 1993) a aorta abdominal desses animais sugeriu um estreitamento aparente no sentido craniocaudal. Achados semelhantes foram constatados em um estudo ultra-sonográfico da aorta abdominal de 131 cães SRD (Kamikawa \& Bombonato 2007). Apesar das escassas referências sobre aparência ultra-sonográfica dos grandes vasos abdominais para espécies primatas (Wagner et al. 2005), para o macaco-prego observou-se a presença da veia cava caudal em íntimo contato com a aorta seguindo ao longo da região ventral das vértebras lombares. $\mathrm{O}$ acesso em decúbito dorsal facilitou a identificação e distinção entre as características da aorta e veia cava caudal. $\mathrm{O}$ fácil acesso à cavidade pélvica tornou possível a visualização detalhada do ponto de bifurcação da veia cava dando origem às veias ilícas.

$\mathrm{O}$ aspecto comparativo da ecogenicidade entre fígado e cortical renal, apresentou-se semelhante ao visualizado em humanos e em cães. Nestes animais, o fígado apresentou ecogenicidade semelhante ou levemente maior do que a cortical renal (Walter et al. 1987, 1988). Os rins de C. apella apresentaram formato uniformemente oval, semelhante ao observado no homem (Schwerk 1983, Netter 1989). A relação cortico-medular foi encontrada preservada em todos os animais, com as pirâmides medulares apresentando-se mais hipoecóicas, triangulares e demonstrando um arranjo regular ao redor do seio renal. Isto também tem sido descrito para anatomia ultra-sonográfica normal do sistema renal em humanos e em cães (Brkljacic et al. 1995, Gaschen et al. 2000). Embora autores tenham observado diferença no comprimento renal entre machos e fêmeas de Marmoset, $C$. jacchus (Wagner et al. 2005), em nossos estudos estes dados não foram estatisticamente significantes.

Embora de fácil visualização no humano (Bates 2004) e no cão (Nyland 2002), o baço foi considerado o órgão de mais difícil visualização, devido ao seu pequeno tamanho, como é observado no gato (Nyland 2002), sendo devidamente avaliado em apenas um caso e não se constituindo uma amostra representativa, não sendo citado neste trabalho.

O parênquima hepático do macaco-prego mostrou-se semelhante às observações feitas para o homem (Bates 2004) e os relatos da literatura para cães e gatos (Partington \& Biller 1996, Nyland 2002). Em alguns momentos este se mostrou hiperecogênico, tal qual também é descrito em répteis e em eqüinos, constituindo-se como um achado normal e observado também em procedimentos pós-anestesia (Pennick et al. 1991, Patterson 1996). A vesícula biliar preservou características conforme citações entre as espécies domésticas (Nyland 2002). Apresentou-se sempre repleta e com conteúdo anecóico, não demonstrando assim, indícios de doença do sistema biliar, bastante comum nas visualizações da vesícula biliar para algumas espécies primatas (Pissinatti et al. 1992), carnívoros (Léveillé et al. 1996) e mesmo no homem (Shea et al. 1994, Pandey et al. 1996, Petroni et al. 2000).

\section{CONCLUSÃO}

O exame ultra-sonográfico mostrou-se como uma técnica eficiente, não-invasiva, rápida e reprodutível para a avaliação da cavidade abdominal em macacos-prego. Muitos achados ultrasonográficos foram similares aos observados na espécie humana e espécies domésticas como cães e gatos. $O$ conhecimento da anatomia ultra-sonográfica normal das estruturas abdominais desses animais permitirão fazer inferências a respeito de condições patológicas, quando existentes, e auxiliar profissionais que militem na área de clínica e cirurgia de animais silvestres.

\section{REFERÊNCIAS}

Bates J.A. 2004. Abdominal Ultrasound: how, why and when. $2^{\text {nd }}$ ed. Churchill Livingstone, London, p.284.

Brkljacic B., Drinkovic I., Delic-Brkljacic D. \& Hebrang A. 1995. Age-related changes of renal vascular resistance in normal native kidneys: colour duplex Doppler ultrasound assessment. Radiol. Oncol. 29:102-106.

Bluth E.I. 1984. Ultrasound of the abdominal aorta. Arch. Intern. Med. 144:377380.

Cerri G.C. \& Rocha D.C. 1993. Ultra-Sonografia Abdominal. Sarvier, São Paulo, p.459.

Coelho N.A. 1993. Ecografia e doppler vascular: aorta abdominal e seus ramos. Revta Angiol. Cir. Vasc. 2:148-157.

Gaschen L., Menninger K. \& Schuurman H.J. 2000. Ultrasonography of the normal kidney in the cynomolgus monkey (Macaca fascicularis): morphologic and Doppler findings. J. Med. Primatol. 29:76-84.

James A.E., Brayton J.B., Novak G., Wight D., Shehan T.K., Bush R.M. \& Sanders R.C. 1976. The use of diagnostic ultrasound in evaluation of the abdomen in primates with emphasis on the rhesus monkey (Macaca mulatta). J. Med. Primatol. 5:160-175.

Kamikawa L. \& Bombonato P.P. 2007. Ultrasonography of abdominal aorta and its branches in dogs. Ciência Rural, Santa Maria, 37:412-417.

Léveillé R., Biller D.S. \& Shiroma J.T. 1996. Sonographic evaluation of the common bile duct in cats. J. Vet. Int. Med. 10:296-299.

Matton J.S. \& Nyland T.G. 2002. Ovaries and uterus, p.231-249. In: Nyland T.G. (ed.), Small Animal Ultrasound. $2^{\text {nd }}$ ed. Saunders Company, Philadelphia.

Netter F.H. 1989. Atlas of Human Anatomy. Ciba Geigy Corp., Japan, p.567-574. 
Nyland T.G., Matton J.S., Hergesell E.J. \& Wisner E.R. 2002. Liver, p.93-127. In: Nyland T.G. (ed.), Small Animal Ultrasound. $2^{\text {nd }}$ ed. Saunders Company, Philadelphia.

Nyland T.G., Matton J.S., Hergesell E.J. \& Wisner E.R. 2002. Spleen, p.129143. In: Nyland T.G. (ed.), Small Animal Ultrasound. $2^{\text {nd }}$ ed. Saunders Company, Philadelphia.

Partington B.P. \& Biller D.S. 1996. Liver, p.105-130. In: Green R. (ed.), Small Animal Ultrasound. Lippincott-Raven, Philadelphia.

Schwerk W.B. 1983. Nieren, p.81-85. In: B. Braun, R. Guenther \& W.B Schwerk (ed.), Ultraschall Diagnostik, Lehrbuch and Atlas. Ecomed, Landsberg, Alemanha.

Patterson M. 1996. Diagnostic aids in equine cardiology, p.70-115. In: Patterson M. (ed.), Equine Cardiology. Oxford Blackwell Science, London.

Pennick D.G., Stewart J.S., Paul-Murphy J. \& Pion P. 1991. Ultrasonography of the California desert tortoise (Xerobates agassizi): anatomy and physiology. Vet. Radiol. 32:112-116.

Pissinatti A., Cruz J.B., Nascimento M.D., Rocha e Silva R. \& Coimbra-Filho A.F. 1992. Spontaneous gallstones in marmosets and tamarins. Fol. Primatol. 59:44-50
Pandey M., Khatri A.K., Sood B.P., Shukla R.C. \& Sukla V.K. 1996. Cholecystosonographic evaluation of the prevalence of gallbladder disease: a university hospital experience. Clin. Imag. 20:269-272.

Petroni M.L., Jazrawi R.P., Pazzi P., Zuin M., Lanzini A., Fracchia M., Facchinetti D., Alvisi V., Ferraris R., Bland J.M., Heaton K.W., Podda M. \& Northfield T.C. 2000. Risk factors for the development of gallstone recurrence following medical dissolution. The British-Italian Gallstone Study Group. Eur. J. Gastroenterol. Hepatol. 12:695-700.

Shea J.A., Berlin J.A., Escarce J.J., Clarke J.R., Kinosian B.P., Cabana M.D., Tsai W.W., Horangic N., Malet P.F. \& Schwartz J.S.1994. Revised estimates of diagnostic test sensitivity and specificity in suspected biliary tract disease. Arch. Intern. Med. 154:2573-2581.

Walter P.A., Feeney D.A., Johnston G.R. \& O'Leary T.P. 1987. Ultrasonographic evaluation of renal parenchymal diseases in dogs: 32 cases (1981-86). J. Am. Vet. Med. Assoc. 191:999-1007.

Walter P.A., Johnston G.R., Feeney D.A. \& O’Brien T.D. 1988. Applications of ultrasonography in the diagnosis of parenchymal kidney disease in cats: 24 cases (1981-86). J. Am. Vet. Med. Assoc. 192:92-98.

Wagner W.M. \& Kirberger R.M. 2005. Transcutaneous ultrasonography of the abdomen in the normal common marmoset (Callithrix jacchus). Vet. Rad. Ultrasound. 46:251-258. 